\title{
Influence of beam radius on deposition parameters in dielectric under high energy electron irradiation
}

\author{
Yinghong Zuo ${ }^{1,2, *}$, Yuan $\mathrm{Wei}^{1,2}$, and Jinhui $\mathrm{Zhu}^{1,2}$ \\ ${ }^{1}$ Northwest Institute of Nuclear Technology, Xi'an, 710024, China \\ ${ }^{2}$ State Key Laboratory of Intense Pulsed Radiation Simulation and Effect, Xi' an 710024, China
}

\begin{abstract}
To study the influence of the electron beam radius on the deposition parameters in dielectric under high energy electron irradiation, a model of dielectric irradiated by plane electron beam source was established. Monte Carlo simulation method was used to calculate the distribution of the injection current density, injection charge density, dose rate and radiation induced conductivity in the dielectric layer of Teflon after electron irradiation with different energies and different beam radii. The results show that the radius of the electron beam source has little influence on the distribution trend of the injection current density in the dielectric layer, as well as the peak position of the injection charge density and the dose rate, but it will significantly affect the peak value of injection charge density and the dose rate. When the electron beam radius is smaller than the dielectric layer radius, the calculated peak is higher than that of the electron beam completely covering the dielectric layer. The higher the electron energy is, the greater the relative deviation will be.
\end{abstract}

\section{Introduction}

When the dielectric materials used in the spacecraft are irradiated by high energy electrons, the electrical charge will be deposited inside the dielectric because of the insulation properties of the dielectric material, and then the deep charging phenomenon is generated in the medium layer[1-3]. After the charging electric field inside the dielectric exceeds the threshold of electric field breakdown, the discharge pulse will be generated, which will endanger the safety of the spacecraft. Therefore, the issue of deep charging of dielectric caused by high energy electron irradiation has attracted the attention of researchers at home and abroad [4-6]. To obtain the charging electric field of dielectric material irradiated by high energy electron beam, it is necessary to obtain the physical parameters such as the distribution of the injection current density, the distribution of the deposited charge and the distribution of the deposition energy in the dielectric, and then the time evolution of the electric field can be obtained through the Poisson's equation [7-8]. There are two kinds of methods to calculate out the parameters of charge deposition and energy deposition. One is to use the analytical method [9-10], the other is to simulate the transport process of high

\footnotetext{
* Corresponding author: zuoyinghong@nint.ac.cn
} 
energy electrons in dielectric materials based on Monte Carlo simulation, and obtain the physical deposition quantities by statistics[11-12].

In the Monte Carlo simulation of high energy electron irradiation process, it is necessary to establish the calculation model of electron irradiation medium. In the simulation model, the irradiated area of the dielectric material is often limited. Because the mass of electron is very small, the electron is easily scattered outside the irradiated area. So in the process of electron irradiation, the electron will expand to the area outside the range of the electron irradiated area, this will lead to the simulation results being affected by the modeling parameters in the established model. In the present research work, most of them only select a specific model parameter to calculate, and the influence of the model parameters on the simulation results has not been reported. To quantitatively study the influence of the radius of the electron beam and the size of the irradiated dielectric material on the simulation results, a Monte Carlo simulation model of Teflon irradiated by high energy electron is established in this paper. The deposition parameters under different radii of electron beam irradiation are obtained and the influence of the electron beam radius on the simulation results is summarized.

\section{Physical model and method}

Figure 1 shows the model of Teflon irradiated by high electron beam. The electron beam is a unidirectional plane source with a radius of $r_{\mathrm{e}}$, the incident direction of electron beam is perpendicular to the surface of Teflon, and the incident position is located directly in front of the dielectric. The whole model is set in vacuum. The shape of Teflon is circular plate with the thickness of $d=0.4 \mathrm{~cm}$ and the radius of $R=0.8 \mathrm{~cm}$. the density of Teflon is 2.2 $\mathrm{g} / \mathrm{cm}^{3}$. In the simulation model, the dielectric of Teflon is divided into 100 layers, and the thickness of each layer is $0.004 \mathrm{~cm}$. The energy of the incident electron is set to be two typical values of $1 \mathrm{MeV}$ and $2 \mathrm{MeV}$, which can represent two cases that the range of electron greater than or less than the thickness of the dielectric. The radius of electron beam source is set to be 5 different values, that are $0.1 \mathrm{~cm}, 0.3 \mathrm{~cm}, 0.5 \mathrm{~cm}, 0.7 \mathrm{~cm}$ and $0.9 \mathrm{~cm}$. The first four radii are smaller than the thickness of the dielectric, and the last radius allows the electron beam to completely cover the dielectric of Teflon.

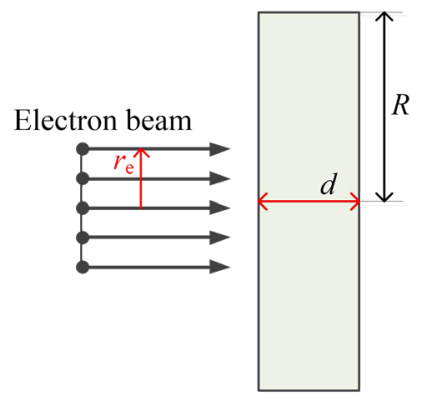

Fig.1. The model of Teflon irradiated by high energy electron beam.

The incident current density is set to be $J_{0}=0.1 \mathrm{pA} / \mathrm{cm}^{2}$ in the simulation. During each irradiation simulation, the electron density of the emitted electrons is the same. The number density of incident electrons is $6.25 \times 10^{5} \mathrm{~cm}^{-2} \mathrm{~s}^{-1}$ according to the incident current density. The Monte Carlo simulation program MCNP 4B is applied to carry out the simulation of electron transport process ${ }^{[13]}$, and the number of simulated electrons is $N_{0}=10^{7}$, which can make the statistical error of all the simulation results of this paper less than $5 \%$. The distribution of injection electron flow $F_{1}(x)$ at different positions $x$ in Teflon layer is calculated by the F1 tally card, and the unit is 1 . The distribution of the deposition charge 
$F_{8}(x)$ with the depth of Teflon is calculated by $+\mathrm{F} 8$ tally card, and the unit is 1 . The distribution of energy deposition $D_{\mathrm{E}}(x)$ in the dielectric layer is calculated by *F8 tally card, and the unit is $\mathrm{MeV}$. The results of $F_{1}(x), F_{8}(x)$, and $D_{\mathrm{E}}(x)$ obtained from Monte Carlo simulation are converted to injected current density $J(x)$, injected charge density $Q(x)$ and dose rate $\dot{D}_{(x)}$. The conversion expressions are as follows.

$$
\begin{gathered}
J(x)=\frac{F_{1}(x) e}{\mathrm{~d} t \cdot A_{0}} \\
\dot{D}(x)=\frac{D_{\mathrm{E}}(x)}{\mathrm{d} t \cdot m}=\frac{D_{\mathrm{E}}(x) \times 10^{6} \times \mathrm{e}}{\mathrm{d} t \cdot \rho \cdot A_{0} \cdot \Delta x} \\
Q(x)=\frac{F_{8}(x) \times \mathrm{e}}{\mathrm{d} t \cdot A_{0} \cdot \Delta x}
\end{gathered}
$$

where, e is the charge quantity of electron; $\mathrm{d} t$ is the unit time, and it is $1 \mathrm{~s}$ in our simulation; $\rho$ is the density of Teflon; $\mathrm{d} x$ is the thickness of the slice when simulated, it is $0.004 \mathrm{~cm}$ in our simulation; $A_{0}$ is the area irradiated by the high energy electron beam, $A_{0}=\pi r_{\mathrm{e}}^{2}$ when the radius $r_{\mathrm{e}}$ of the electron beam is less than the radius $R$ of Teflon, $A_{0}=\pi R^{2}$ when the radius $r_{\mathrm{e}}$ of the electron beam is greater than the radius $R$ of Teflon, in which case the dielectric of Teflon is completely irradiated by electron beam.

The expression of the radiation induced conductivity of Teflon under high energy electron irradiation is

$$
\sigma(x)=\sigma_{0}(x)+\sigma_{\mathrm{e}}(x)=\sigma_{0}(x)+k_{\mathrm{p}} \dot{D}(x)^{\Delta}
$$

where, $\sigma_{0}(x)$ is the intrinsic conductivity when it is not irradiated, the value is $1.0 \times 10^{-16} \mathrm{~S} / \mathrm{m}$ for Teflon; $\dot{D}(x)$ is the dose rate due to electron irradiation, and the unit is $\mathrm{rad} / \mathrm{s} ; k_{\mathrm{p}}$ and $\triangle$ are coefficients associated with the dielectric material, for Teflon, $\mathrm{kp}=1.25 \times 10^{-14}$ $(\mathrm{S} / \mathrm{m}) /(\mathrm{rad} / \mathrm{s})$, and $\triangle=0.65$ for Teflon in this paper.

\section{Simulation result and discussion}

Based on the above model, we get the charge and energy deposition distribution of high energy electrons in Teflon dielectric.

\subsection{The case of electron range is less than thickness of dielectric}

Figure 2 is the simulation result for the case of the electron energy $1 \mathrm{MeV}$. The literature [11] used Geant 4 and obtained the dose rate and deposition charge distribution of $1 \mathrm{MeV}$ electrons in the Teflon with the size of $4 \mathrm{~mm} * 4 \mathrm{~mm} * 4 \mathrm{~mm}$. The peak of dose rate is at the depth of $0.65 \mathrm{~mm}$ in the dielectric material. In our paper, the peak of dose rate simulated by using MCNP4B program is at the depth of $x=0.78$ in Teflon. It is basically consistent with the results in the literature [11]. From the simulation results of figure 2(a), it is known that the electrons with energy of $1 \mathrm{MeV}$ have not completely penetrated the Teflon with a thickness of $4 \mathrm{~mm}$, and there is an unirradiated area in the dielectric. The injected current density $J(x)$ gradually decreases to zero with the increase of the dielectric depth, while the deposition charge density and dose rate increase firstly and then gradually decrease to zero with the increase of the depth of the dielectric. The peak value of the injected current density and the dose rate appeared in $x=1.5 \mathrm{~mm}$ and $x=0.78 \mathrm{~mm}$ of Teflon dielectric, respectively.

From the simulation results shown in figure 2, it can be seen that the curve of injected current density varies with the depth of the dielectric basically coincides at different 
electron beam radii, which indicates that the radius of the electron beam has little effect on the distribution trend of the injection current density. The position of the peak value of the deposition charge remains unchanged at the different radius of the electron beam, all of which are at depth of $x=1.5 \mathrm{~mm}$, but the peak values are unequal in different cases. The peak value of the deposited charge is $-7.19 \times 10^{-13} \mathrm{C} / \mathrm{cm}^{3}$ when the radius of electron beam $r_{\mathrm{e}}$ is greater than the radius of Teflon dielectric $R$, while that is $-7.87 \times 10^{-13} \mathrm{C} / \mathrm{cm}^{3}$ when $r_{\mathrm{e}}<R$, which is $9.5 \%$ higher on the basis of the case of the electron beam covering the dielectric layer when $r_{\mathrm{e}}<R$.
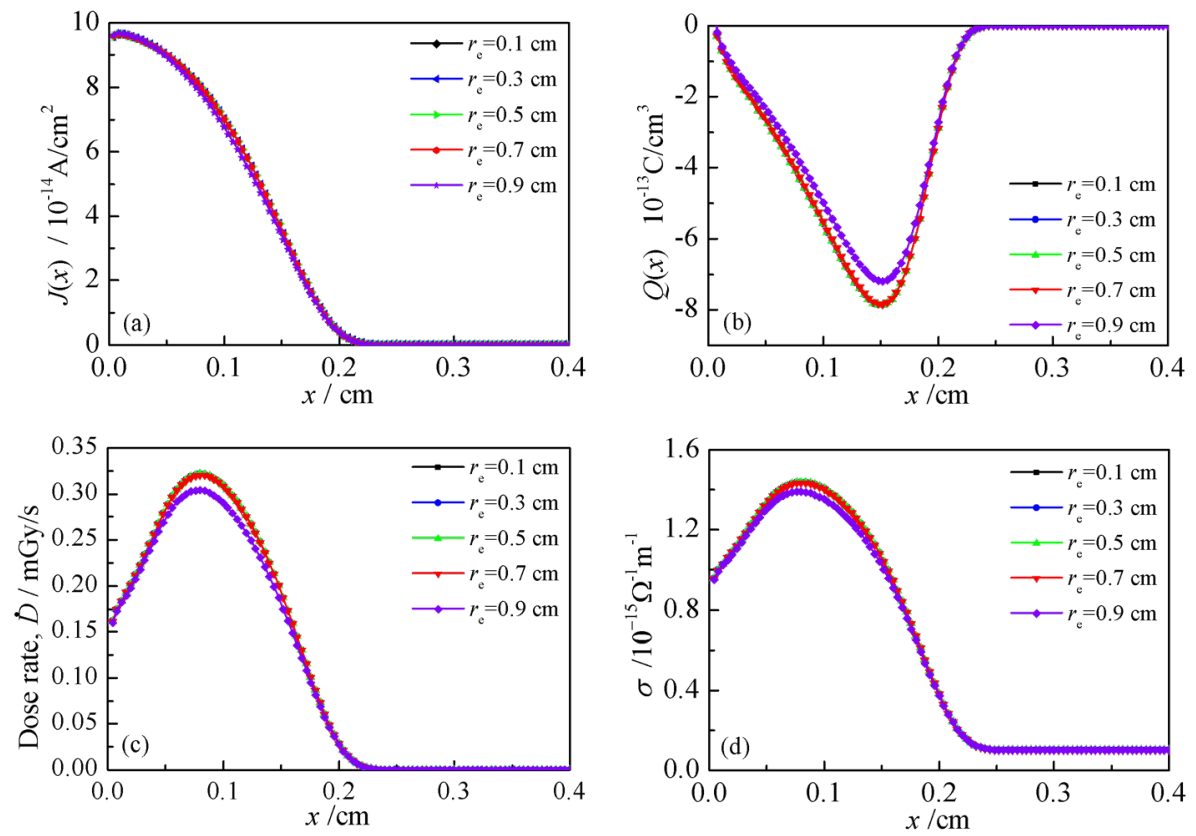

Fig. 2. Simulation results for the case of electron with energy of $1 \mathrm{MeV}$. (a) Injected current density, (b) Injected charge density, (c) Dose rate, (d) Radiation induced conductivity.

The dose rate in figure 2(c) is calculated out from the distribution of deposition energy. The results show that the peak vale of dose rate all appears at $x=0.78 \mathrm{~mm}$ when the electron beam radius is different. The peak values of the dose rate for the cases of $r_{\mathrm{e}}>R$ and $r_{\mathrm{e}}<\mathrm{R}$ are $0.30 \mathrm{mGy} / \mathrm{s}$ and $0.32 \mathrm{mGy} / \mathrm{s}$, respectively, and the relative deviation between them is $6.7 \%$. The radiation induced conductivities corresponding to the peak dose rates are $1.386 \times 10^{-15}$ $\mathrm{S} / \mathrm{m}$ and $1.441 \times 10^{-15} \mathrm{~S} / \mathrm{m}$, respectively, and the relative deviation between them is $3.9 \%$. It can be seen that when the energy of the electron beam is $1 \mathrm{MeV}$, the radius of the electron beam source has little influence on the distribution trend of the injected current density varies with the depth of the dielectric, and has no effect on the position of the peak value of the deposited charge and the deposition energy. However, when the radius of the electron beam is greater than the radius of the dielectric, the peak value of the deposited charge and the peak value of the deposition energy are both less than that of the case of the radius of the electron beam less than the radius of the dielectric.

\subsection{The case of electron range is greater than the thickness dielectric}

Figure 3 shows the simulation result for the case of the electron energy $2 \mathrm{MeV}$. It can be seen that the electron with energy of $2 \mathrm{MeV}$ has completely penetrated the dielectric layer with the thickness of $4 \mathrm{~mm}$. In fact, the range of electron with energy of $2 \mathrm{MeV}$ in the 
Teflon dielectric is $R_{\mathrm{s}}=0.446 \mathrm{~cm}$, which is greater than the thickness of Teflon dielectric. Figure 3(a) shows that the curve of injected current density varies with the depth of the dielectric basically coincides when $r_{\mathrm{e}} \leq 0.5 \mathrm{~cm}$. The calculation results of injected current density $J(x)$ for the case of $r_{\mathrm{e}}=0.7 \mathrm{~cm}$ is less than that for the case of $r_{\mathrm{e}} \leq 0.5 \mathrm{~cm}$, and when the radius of the electron beam radius $r_{\mathrm{e}}>R$, the injected current density is further reduced. Therefore, it is concluded that when the difference between the radius of the dielectric layer and the radius of the electron beam is more than 0.5 times the electron range, that is, $R$ $r_{\mathrm{e}}>0.5 R_{\mathrm{s}}$, the radius of the electron beam has little effect on the calculation results of the injected current density.

As can be seen from figure 3(b) $\sim 3(\mathrm{~d})$, when $r_{\mathrm{e}} \leq 0.5 \mathrm{~cm}, r_{\mathrm{e}}=0.7 \mathrm{~cm}$ and $r_{\mathrm{e}}=0.9 \mathrm{~cm}$, the positions of the peak value of the deposited charge are all at $x=0.34 \mathrm{~cm}$, and the peak values of the deposited charge are $-3.89 \times 10^{-13} \mathrm{C} / \mathrm{cm}^{3},-3.58 \times 10^{-13} \mathrm{C} / \mathrm{cm}^{3},-3.18 \times 10^{-13} \mathrm{C} / \mathrm{cm}^{3}$, respectively. On the basis results of the case of $r_{\mathrm{e}}>R$, the relative deviations are $22.2 \%$ and $12.6 \%$, respectively. When $r_{\mathrm{e}} \leq 0.5 \mathrm{~cm}, r_{\mathrm{e}}=0.7 \mathrm{~cm}$ and $\mathrm{re}=0.9 \mathrm{~cm}$, the positions of the peak value of the dose rate are all at $x=0.18 \mathrm{~cm}$, and the peak values of the dose rate are 0.287 $\mathrm{mGy} / \mathrm{s}, 0.276 \mathrm{mGy} / \mathrm{s}$, and $0.255 \mathrm{mGy} / \mathrm{s}$, respectively. With the results of the case of $r_{\mathrm{e}}>R$ as the basis, the relative deviations for cases of $r_{\mathrm{e}} \leq 0.5 \mathrm{~cm}$ and $r_{\mathrm{e}}=0.7 \mathrm{~cm}$ are $12.5 \%$ and $8.2 \%$, respectively. The peak values of the radiation induced conductivity are $1.34 \times 10^{-15} \mathrm{~S} / \mathrm{m}$, $1.31 \times 10^{-15} \mathrm{~S} / \mathrm{m}$, and $1.25 \times 10^{-15} \mathrm{~S} / \mathrm{m}$, respectively; and the relative deviations for cases of $r_{\mathrm{e}} \leq 0.5 \mathrm{~cm}$ and $r_{\mathrm{e}}=0.7 \mathrm{~cm}$ are $7.2 \%$ and $4.8 \%$, respectively.
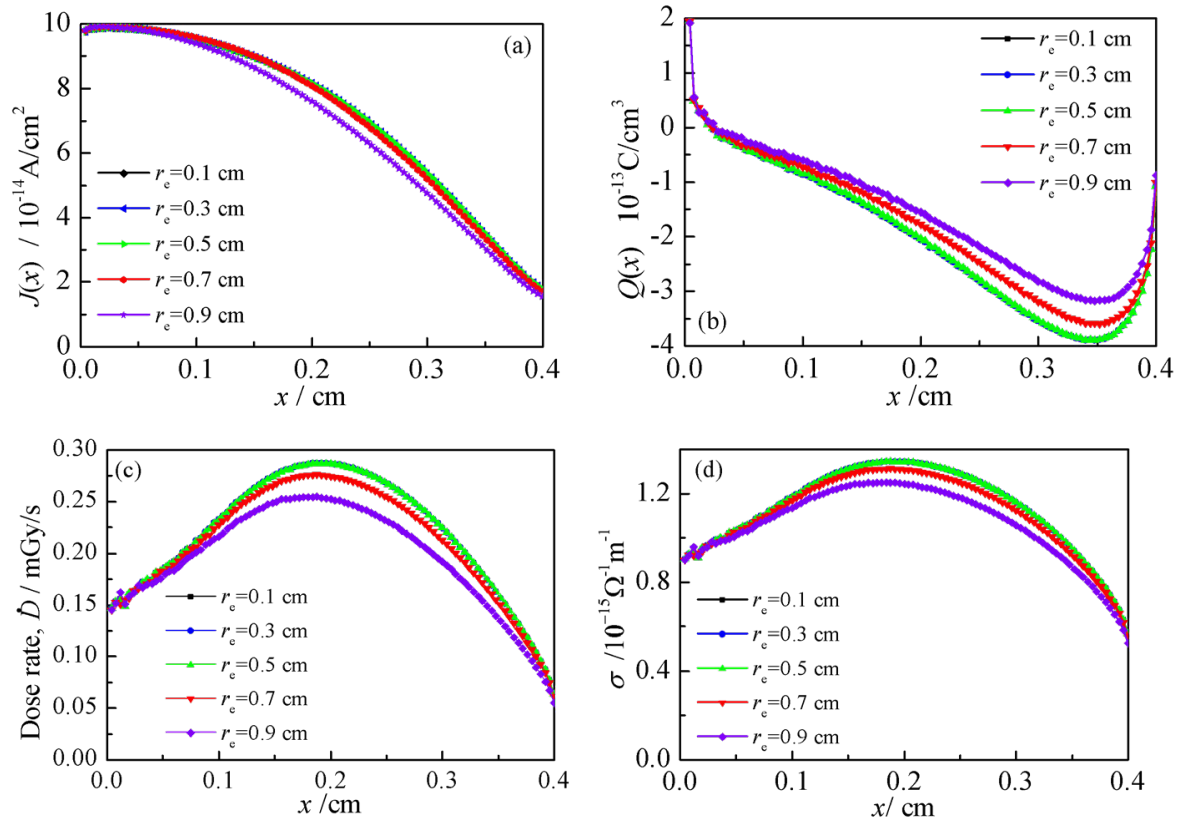

Fig. 3. Simulation results for the case of electron with energy of $2 \mathrm{MeV}$. (a) Injected current density, (b) Injected charge density, (c) Dose rate, (d) Radiation induced conductivity.

Compared with the results of figure 2 and figure 3, it can be seen that, under the same condition of $R-r_{\mathrm{e}}$, the higher the energy of electron is, the greater the relative deviation between the calculated results under the $r_{\mathrm{e}}<R$ condition and the calculated results under the $r_{\mathrm{e}}>R$ condition is. Considering the actual situation, the dielectric of the spacecraft may be completely irradiated by high energy electrons, so the radius of the electron beam source should be properly larger than the size of the irradiated dielectric sample in order to make the simulation model more reasonable and reliable. 


\section{Conclusions}

In this paper, the physical calculation model of the Teflon dielectric irradiated by high energy electron beam was established. By using Monte Carlo simulation method, the distribution of the injected current density, deposition charge density, and the dose rate in the dielectric are obtained at various cases of irradiation electron beams with different energy and different beam radii. The results show that the radius of the electron beam does not affect the variation trend of the calculated distribution of the deposition parameters in the dielectric layer, and has little influence on the position of the peak value of the calculated physical deposition parameters, but it will affect the amplitude of the peak value. When the difference between the radius of the electron beam and the radius of the irradiated dielectric is greater than 0.5 times the range of the electron, the radius of the electron beam has little influence on the calculated injected current density, and the higher the energy of the electron is, the more significant the effect of the electron beam radius on the calculation result. Considering that the spacecraft or satellite devices are "immersed" in the electronic environment in the space radiation environment, the radius of the electron beam is recommended to be larger than the radius of the irradiated dielectric in simulation.

\section{Acknowledgments}

Supported by State Key Lab of Intense Pulsed Radiation Simulation and Effect Basic Research Foundation (No.SKLIPR1504).

\section{References}

1. J.G. Huang, D. Chen, Acta Physics Sinica, 53(3), 961-966 (2004)

2. N.W. Green, J.R. Dennison, IEEE Transactions on Plasma Science, 36(5), 2482-2490 (2008)

3. X.Q. Yu, H.F. Chen, Q.G. Zong, J.Z. Wang, W.H. Shi, H. Zou, J.Q. Zou, W.Y. Zhong, Z. Chen, S.P. Shao, X.H. Jia, IEEE Transactions on Nuclear Science, 64(11), 28222828 (2017)

4. R.H. Quan, Z.L. Zhang, J.W. Han, J.G. Huang, X.J. Yan, Acta Physica Sinica, 58(2), 1205-1210 (2009).

5. S. Li, G. Li, Chinese Science Bulletin, 62(10), 990-1003 (2017)

6. R. Renoud, F. Mady, J.P. Ganachaud, Journal of Physics: Condensed Matter, 14(2), $231(2001)$

7. J.G. Huang, D. Chen, L.Q. Shi, Chinese Journal of Space Science, 24(5), 346-353 (2004)

8. G. Wilson, J.R. Dennison, A.E. Jensen, J. Dekany, IEEE Transactions on Plasma Science, 41(12), 3536-3544 (2013)

9. J.G. Huang, D. Chen, Chinese Journal of Geophysics, 47(3), 392-397 (2004)

10. S.T. Li, G.C. Li, D.M. Min, N. Zhao, Acta Physics Sinica, 62(5), 059401 (2013)

11. X.G. Qin, D.Y. He, J. Wang, Acta Physica Sinca, 58(1), 684-689 (2009)

12. S.L. Roy, F. Baudoin, V. Griseri, C. Laurent, G. Teyssedre, Journal of Physics D: Applied Physics, 43(31), 315402 (2010)

13. J. F. Briesmeister, MCNP-A general Monte Carlo N-particle transport code, Version $4 B$, LA-12625-M, (Los Alamos National Laboratory, New Mexico, 1997) 